\title{
Recombinant human bone morphogenetic protein-2-induced ossification of the ligamentum flavum in rats and the associated global modification of histone $\mathrm{H} 3$
}

\author{
Laboratory investigation
}

\author{
Xiao-Fei Hou, Ph.D., Dong-Wei Fan, Ph.D., Chui-Guo Sun, M.D., \\ and Zhong-Qiang Chen, M.D. \\ Department of Orthopedics, Peking University Third Hospital, Beijing, China
}

\begin{abstract}
Object. The primary object of this investigation was to study recombinant human bone morphogenetic protein-2 (rhBMP-2)-induced ossification of the ligamentum flavum and associated histone $\mathrm{H} 3$ modification in a rat model. In an additional set of studies the authors investigated spinal cord and behavioral changes in the same model.

Methods. The authors report on 2 separate sets of studies. A total of 90 rats were used for the 2 sets of studies (45 each); in each study, a lyophilized rhBMP-2 and collagen mixture (20 $\mu \mathrm{g}$ rhBMP-2 and $200 \mu \mathrm{l}$ collagen) was implanted in the lumbar extradural space in 18 rats; another 18 animals were used for a sham-operation control group and underwent implantation of lyophilized collagen without rhBMP-2 at the same level; an additional 9 animals were used as untreated controls. Lumbar spinal samples were harvested from the rhBMP-2 groups and the shamoperation control groups at 1 week, 3 weeks, and 9 weeks after the operation. Samples were also obtained from untreated controls at the same time points. All samples were scanned using micro-CT and then made into paraffinembedded sections. The sections from the first set of 45 rats were stained using elastica van Gieson and toluidine blue, and the expression of histone modifications (H3K9ac, H3K18ac, H3K4me3, and H3K36me3) and osteogenic transcription factors (osterix, Runx2) was detected by immunohistochemistry. In the second set of studies, hindlimb motor function was assessed at 1 week, 3 weeks, and 9 weeks after surgery. After behavioral evaluation, samples were harvested, scanned using micro-CT, and then made into paraffin-embedded sections. The sections were stained using Luxol fast blue. The expression of NeuN was also detected using immunohistochemistry.

Results. Ossification was seen in the rhBMP-2 group from 1 week after insertion, and the volume of ossified mass increased at 3 and 9 weeks. There was no ossification seen in the sham-surgery and normal controls. The pathological changes of ossification involved ligament degeneration, cartilage formation, and, finally, bone replacement. Spinal cord evaluation showed a significant decrease in white matter content and number of neurons at 9 weeks after operation in the rhBMP-2-treated group (compared with findings in the sham-surgery and control groups as well as findings at the earlier time points in the rhBMP-2 group).

Using immunohistochemical staining, histone modifications (H3K9ac, H3K18ac, H3K4me3, and H3K36me3) and osteogenic transcription factors (osterix, Runx2) all were found to be expressed in the fibrocartilage area of the rat ossified ligamentum flavum samples (rhBMP2 group).

Conclusions. This rhBMP-2-induced OLF is a typical endochondral ossification, which is similar to clinical OLF. The compressed spinal cord around the ossification site showed signs of a chronic degenerative process. Histone $\mathrm{H} 3$ modifications (H3K9ac, H3K18ac, H3K4me3, and H3K36me3) may play an important role in OLF. (http://thejns.org/doi/abs/10.3171/2014.4.SPINE13319)
\end{abstract}

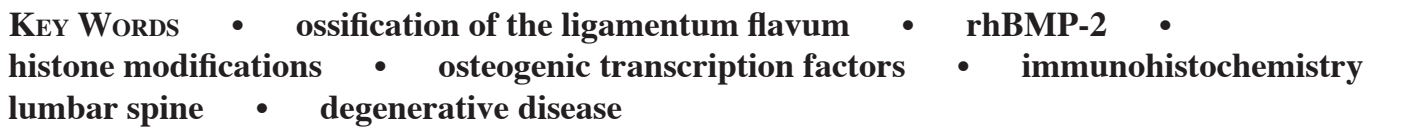

$\mathrm{O}$ SSIFICATION of the ligamentum flavum (OLF), which mainly affects the lower thoracic spine, is the primary cause of thoracic spinal canal stenosis and myelopathy. ${ }^{7}$ This ectopic ossification has a high

Abbreviations used in this paper: $\mathrm{EVG}=$ elastica van Gieson; $\mathrm{LFB}=$ Luxol fast blue; OLF = ossification of the ligamentum flavum; $\mathrm{PBS}=$ phosphate-buffered saline; rhBMP-2 = recombinant human bone morphogenetic protein-2 prevalence in Japanese and Chinese populations. Our previous epidemiological survey showed a standardized prevalence rate of $63.9 \%$ in Chinese people. ${ }^{14}$ In the past several years, extensive studies have investigated the mechanism of OLF at the histopathological and cellular levels. ${ }^{5,11,19,29,30}$ A series of crucial factors have been identified. However, the effect of these factors has not been investigated in vivo, due to the lack of a proper OLF animal model similar to clinical OLF.

Epigenetics is the study of the events that regulate 


\section{RhBMP-2-induced OLF and associated histone modifications}

gene activity without changing the underlying DNA sequence. DNA methylation and histone posttranslational modifications are the 2 major mechanisms of epigenetic gene regulation. ${ }^{9}$ Several studies have shown that patterns of histone modification have been associated with many essential biological processes, such as stem cell maintenance and differentiation. ${ }^{10,16,21}$ Previous studies have revealed that mesenchymal stem cells exist in ligamentum flavum and play a crucial role in the development of OLF. ${ }^{3,18}$ Whether histone modifications are involved in this ossification process has not been studied.

In this study, we investigated the pathological features of a rat OLF model induced by recombinant human bone morphogenetic protein-2 (rhBMP-2) which is known for its strong osteoinductive effect, ${ }^{13}$ and the expression of 4 histone $\mathrm{H} 3$ modifications during this process. In addition, another set of rats was used to assess possible behavioral changes and the pathological features of the compressed spinal cord.

\section{Methods}

\section{Animals}

A total of 90 adult male Sprague-Dawley rats (8 weeks old, $\sim 200 \mathrm{~g}$ ) were used for this study, which included two separate sets of experiments. For each set of experiments, there were 18 rats in a rhBMP-2-treated group, 18 in a sham-operation control group, and 9 in a normal control group. This study was approved by the Ethics Committee of the Peking University Health Science Centre.

\section{Implant Materials}

Recombinant human bone morphogenetic protein-2 was purchased from Sino Biological Inc. A collagen solution (Cellmatrix Type I-A; Nitta Gelatin Inc.) was used as the rhBMP-2 carrier to maintain continuous-release of rhBMP-2. Twenty micrograms of rhBMP-2 was dissolved in $200 \mu \mathrm{l}$ of collagen solution, and the mixture was lyophilized (Modulyod-230; Thermo Electron Corp.) on a sheet. For use in the sham-operation control groups, an equal volume of collagen solution, without rhBMP-2, was lyophilized.

\section{Operative Procedure}

The operation was performed as previously described with several modifications. ${ }^{17}$ Briefly, anesthesia was induced with an intraperitoneal injection of pentobarbital sodium $(5.0 \mathrm{mg} / 100 \mathrm{~g}$ body weight). Following disinfection of the surgical site, a longitudinal skin incision was made in middle of the rat's back over L1-2 or L2-3. The paraspinal muscles were detached. To obtain a better surgical field, the spinous processes were partially resected, and the adjacent supraspinous and interspinous ligaments were removed. The exposed ligament flavum was split in the middle, and 2 pieces of the lyophilized rhBMP- 2 and collagen (each containing $10 \mu \mathrm{g}$ of rhBMP-2 and $100 \mu \mathrm{l}$ of collagen) were inserted into the posterolateral epidural space (one piece on each side). The paraspinal muscles were sutured, and the skin was closed. In the sham-op- eration control group, the rats were treated with the same operation, except that only lyophilized collagen was implanted in the epidural space.

\section{Behavioral Evaluation}

Hindlimb motor function of was assessed by means of the Basso, Beattie, and Bresnahan locomotor rating scale $(\mathrm{BBB} \text { scale })^{2}$ in the second set of 45 animals at 1 week, 3 weeks, and 9 weeks after the operation. Briefly, rats were allowed to move freely in a circular field for 4 minutes while the movements of the hindlimbs were closely observed. The hindlimb movement was scored by 2 independent observers who were blinded to the experimental design.

\section{Micro-CT Analysis}

In each of the 2 sets of experiments, specimens were harvested from 6 rats in the experimental group at each of 3 time points $-1,3$, and 9 weeks after the operation; in the sham-operation and normal control groups, batches of 6 rats and 3 rats, respectively, were sacrificed at the same time points. Thus for each time point, data were obtained from 12 animals that had undergone rhBMP-2 insertion, 12 that had undergone sham surgery, and 6 normal controls. The animals were randomly selected and were killed with a lethal dose of pentobarbital sodium and transcardially perfused with $4 \%$ paraformaldehyde in phosphatebuffered saline. Next, the entire thoracolumbar spine was removed with the paravertebral muscles.

To quantitatively evaluate the extent of compression of the spinal canal, the operated and adjacent spinal segments were scanned using the micro-CT scanner (Inveon, Siemens Medical Solutions). The specimens were scanned at a resolution of $14 \mu \mathrm{m}(80 \mathrm{kV}, 500 \mu \mathrm{A})$ and were analyzed using Inveon Research Workplace software (Version 3.0, Siemens Medical Solutions). For each animal, the anteroposterior canal diameter at the level of maximum compromise was compared with the anteroposterior canal diameter at the adjacent normal levels above and below the operation site based on axial images from micro-CT. The normal canal anteroposterior diameter was measured at the midvertebral body level. The following equation was used to quantify the maximum compression ratio: ${ }^{15}$

\section{Maximum compression ratio $(\%)=\left[1-\left(\frac{D}{(D u+D d) / 2}\right)\right] \times 100 \%$}

where $\mathrm{D}$ is the anteroposterior canal diameter at the level of maximum protrusion, $\mathrm{Du}$ is the anteroposterior canal diameter at the nearest normal level above the level of operation, and Dd is the anteroposterior canal diameter at the nearest normal level below the level of operation.

\section{Tissue Preparation and Staining}

The entire thoracolumbar spine with the paravertebral muscles for each animal was fixed with $10 \%$ neutral buffered formalin for 48 hours at $4^{\circ} \mathrm{C}$. Next, these samples were decalcified with $10 \%$ ethylenediaminetetraace- 
tic acid (EDTA) for $5-10$ days at $4^{\circ} \mathrm{C}$. The specimens were embedded in paraffin using standard procedures. Serial 4- $\mu$ m-thick sagittal sections from samples from the first set of 45 animals (obtained at the specified time points) were prepared for elastica van Gieson (EVG) and toluidine blue staining to detect pathological changes of the operated ligamentum flavum. Samples from the second set of 45 animals were used for spinal cord assessment: 8 - $\mu$ m-thick transverse sections were obtained from the epicenter of the compression site and prepared for Luxol fast blue (LFB) staining to detect myelin damage. The spared white matter was measured with Image-Pro Plus 5.0 software (Cybernetics). The area of spared white matter was expressed as the percentage of LFB-positive area in the total area of the spinal cord.

\section{Immunohistochemical Analysis}

Serial 5- $\mu$ m-thick sections were cut from the paraffin-embedded specimens for immunohistochemical staining. Sections obtained from the first set of 45 animals ( 6 from the rhBMP-2 group, 6 from the sham surgery group, and 3 normal controls at each time point) were used to examine the expression of histone modifications and osteogenic transcriptional factors. Sections from the second set (also 6 from the rhBMP-2 group, 6 from the sham surgery group, and 3 normal controls at each time point) were used to examine the expression of NeuN. The sections were deparaffinized with xylene and dehydrated in serially graded ethanol solutions. After washing in distilled water, the sections were treated with a $0.3 \%$ $\mathrm{H}_{2} \mathrm{O}_{2}$ solution dissolved in absolute methanol at $20^{\circ} \mathrm{C}$ for 10 minutes and were rinsed with phosphate-buffered saline (PBS, pH 7.4). Antigen retrieval was performed by heating for 30 minutes in a microwave. The sections were then incubated with the following primary rabbit or mouse antibodies (from Abcam Inc. and Millipore) at $4^{\circ} \mathrm{C}$ overnight in a humidified chamber: a polyclonal rabbit anti-rat antibody for acetyl histone H3 Lys9 (H3K9ac) (catalog number: ab10812, 1:100); monoclonal rabbit anti-rat antibody for acetyl histone H3 Lys18 (H3K18ac) (catalog number: ab40888, 1:100); polyclonal rabbit antirat antibody for trimethyl histone H3 Lys4 (H3K4me3) (catalog number: ab8580, 1:200); polyclonal rabbit antirat antibody for trimethyl histone H3 Lys36 (H3K36me3) (catalog number: ab9050, 1:500); polyclonal rabbit antirat antibody for Runx2 (catalog number: ab23981, 1:100); polyclonal rabbit anti-rat antibody for Sp-7/osterix (catalog number: ab22552, 1:200); and monoclonal mouse anti-rat antibody anti-NeuN (catalog number: MAB 377, 1:800). After washing with PBS 3 times for 5 minutes, the sections were reacted with goat anti-rabbit (PV-6001) or goat anti-mouse (PV-6002) immunoglobulin antibodies conjugated to peroxidase-labeled polymer (PowerVison TM, ZSGB-BIO) in a humidified chamber for $30 \mathrm{~min}$ utes at room temperature. After rinsing with PBS at $\mathrm{pH}$ 7.4 , antibody binding was visualized by incubating with DAB solution (Sigma) and the sections were washed in water to remove excess DAB. The sections were counterstained with hematoxylin for visualization of nuclei. The negative control sections were incubated with PBS instead of the primary antibodies using the same condi- tions. To perform a quantitative analysis of NeuN-positive neurons, the average number of NeuN-positive neurons was counted in 5 fields within gray matter in each section by 2 independent observers under a light microscope $(40 \times)$. The results were expressed as average number of NeuN-positive neurons per field in each group.

\section{Statistical Analysis}

SPSS version 13.0 was used for the statistical analysis. For comparison of groups over time, we used 2-way ANOVA followed by the post hoc Bonferroni test. For comparison of simple effects, 2-way ANOVA with post hoc Tukey's analysis was performed. All data were presented as the mean \pm standard error with $p<0.05$ being significant.

\section{Results}

\section{Absence of Paralysis}

None of the 45 rats used for the behavioral evaluation showed any obvious hindlimb motor abnormality or paralysis during the 9-week observation period, and all 45 animals had a full BBB score of 21 .

\section{RhBMP-2-Induced Ossification of the Ligamentum Flavum}

In all of the rats that received the lyophilized rhBMP-2/collagen mixture, the micro-CT images the newly formed bone started from laminae in the operated segment and protruded into the vertebral canal. Ossification initially developed along the ventral surface of the ligamentum flavum. At 9 weeks after insertion of the rhBMP-2/collagen mixture, the dorsal part of the ligamentum flavum was ossified (Fig. 1A).

The diameter was measured (Fig. 1B), and the maximum compression ratio was calculated with the formula shown above for the rhBMP-2-treated group. The mean maximum compression ratio was $13.08 \%( \pm 1.00 \%)$ at 1 week, $23.92 \%( \pm 1.32 \%)$ at 3 weeks, and $46.08 \%( \pm$ $1.42 \%)$ at 9 weeks. There was a significant difference between the 3 time points in the experimental group (p $<0.001$ ) (Fig. 1C), suggesting that the volume of newly formed bone increased over time.

In the sham-surgery and normal control groups, micro-CT showed no abnormal protrusion at the surgical level or corresponding level in the normal controls.

\section{RhBMP-2-Induced OLF in Rats: Typical Endochondral Ossification}

Macroscopically, the protrusion in the spinal canal increased gradually after the operation in the rhBMP-2treated group, and the spinal cord was compressed with obvious distortion at 3 weeks and 9 weeks (Fig. 2A). However, in the control groups, there were no spinal cord deformities.

Elastica van Gieson and toluidine blue staining was used to investigate histomorphological changes of the ligamentum flavum and fibrocartilage. In the normal controls, the intact structure of the ligamentum flavum was uniformly arranged and attached to the craniad and cau- 
A

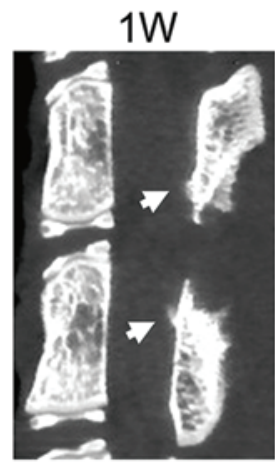

B

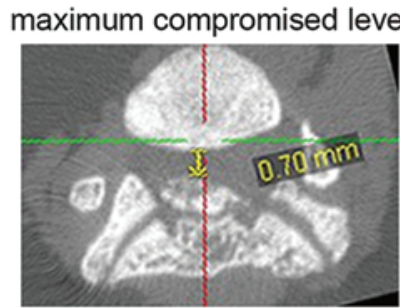

rhBMP-2 treated
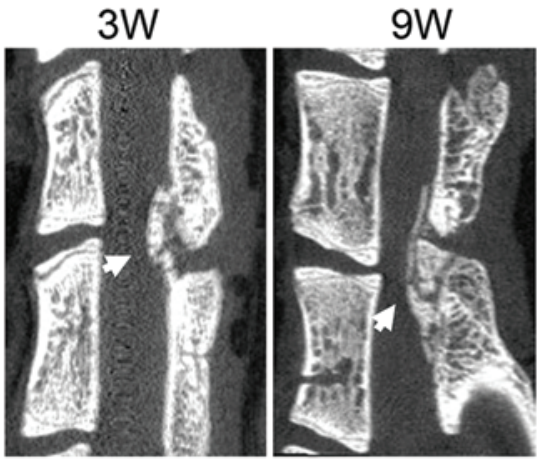

normal level

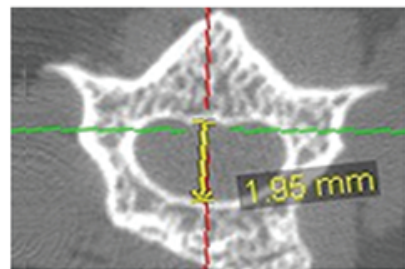

C

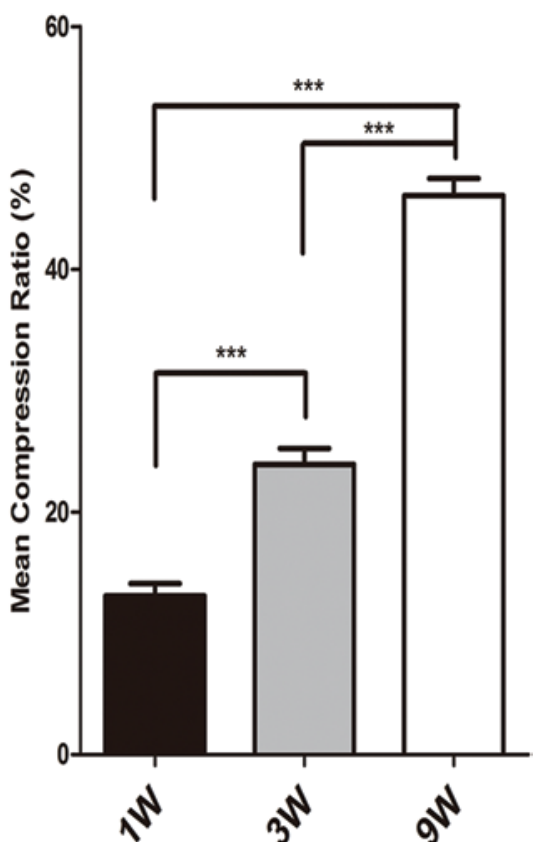

Fig. 1. Analysis of development of OLF in the rhBMP-2-treated group as demonstrated by micro-CT. A: Midsagittal sections obtained from the rat OLF model at 1 week (1w), 3 weeks $(3 w)$, and 9 weeks $(9 w)$ after the operation, illustrating that the ossification started at the insertion site (1w, arrow) in the ligamentum flavum, developed along the ventral surface ( $3 \mathrm{w})$, and finally developed in the dorsal area (9w). B: Axial micro-CT images showing the diameter measurements at the levels of maximum compromise and a normal level. C: Graph showing the increase in the mean maximum compression ratio over time in the rhBMP-2-treated group. The mean compression ratio was calculated from measurements obtained in 12 animals at each time point. ${ }^{* *} p<0.001$.

dad vertebral laminae (Fig. 2B). Newly formed fibrocartilage or bone was not found between the vertebral laminae (Fig. 2C). In the samples from the sham-surgery control group, similar results were observed (Fig. 2B and C).

In the rhBMP-2-treated group, different pathological results were observed. In the samples obtained 1 week after surgery, the arrangement of the ligament was irregular, and the elastic fibers were decreased in number relative to samples from the sham-surgery group and normal controls (Fig. 2B). Newly formed cartilaginous tissues appeared within the ligament (Fig. 2C). A small amount of newly formed bone appeared adjacent to the lamina.
A

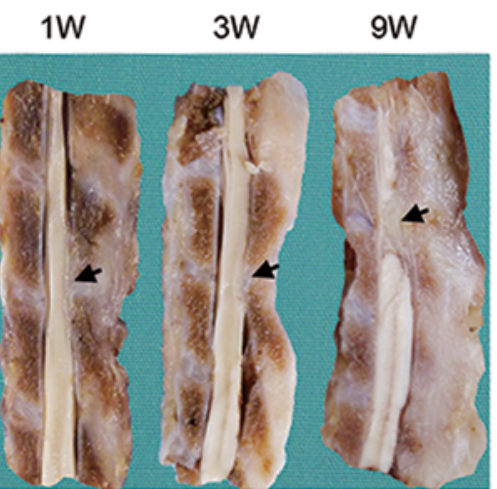

B

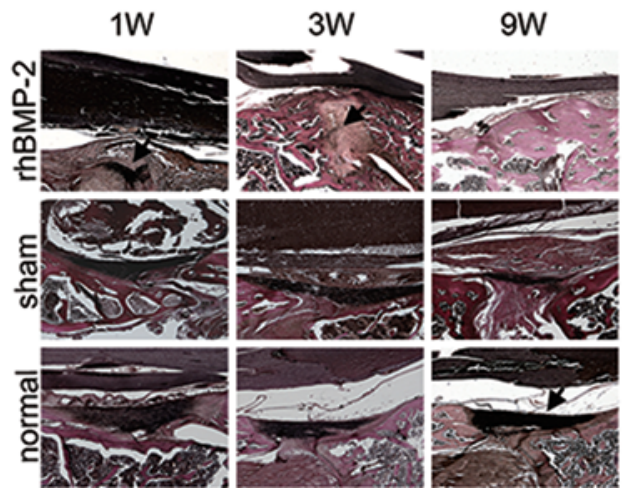

C

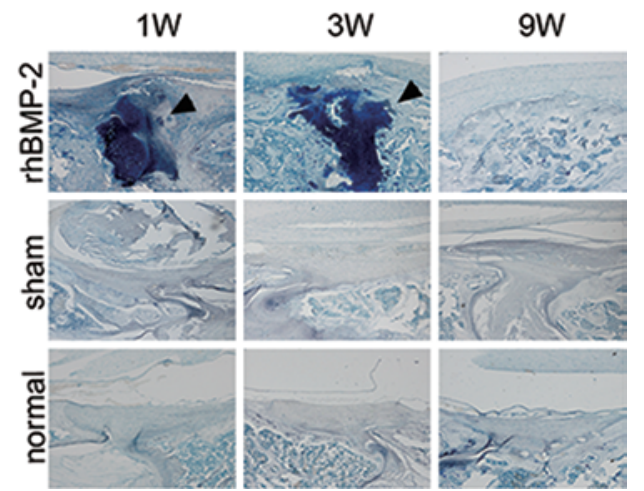

FIG. 2. Representative images of the rhBMP-2-induced OLF and photomicrographs of sections stained with EVG and toluidine blue. A: Midsagittal sections obtained from the rats in the rhBMP-2 group at 1 week (1w), 3 weeks (3w), and 9 weeks $(9 w)$ after surgery. Note that the protrusion (arrow) in the canal increased gradually and the spinal cord became distorted. B: Photomicrographs of sections stained with EVG showing that the structure of ligamentum flavum became increasingly irregular, decomposed (arrows in $1 w$ and $3 w$ images) during ossification, and had completely disappeared by 9 weeks in the rhBMP-2 group. The ligamentum flavum in the normal and sham-operation control groups was intact and dense (arrow). Original magnification $\times 40$. C: Photomicrographs of toluidine blue-stained sections showing that a fibrocartilaginous mass was found between the upper and lower laminae at 1 week and 3 weeks after the operation (arrowhead). The mass was totally replaced at 9 weeks. Fibrocartilaginous tissue was not observed in the normal or sham-operation control groups. Original magnification $\times 40$. 
In the samples obtained 3 weeks after surgery, the ventral layer of the ligamentum flavum had been replaced by fibrocartilage and bone (Fig. 2C). A few irregular collagen fibers remained on the dorsal surface (Fig. 2B). The volume of newly formed bone was increased.

In the samples obtained 9 weeks after surgery, the ligamentum flavum and fibrocartilage tissue between the laminae had disappeared. They had been replaced with bone. The spinal cord compression was more increased (Fig. 2B and C).

\section{Histopathological Changes in Affected Spinal Cord}

The myelin content of spinal cord sections from the operated levels and corresponding levels in normal controls was determined by LFB staining. In both the normal and sham-operation control groups, the extent of LFBpositive staining of the myelin did not decrease over time.
In the compressed epicenter of cord sections from the rhBMP-2-treated group, deformation of the cord increased gradually, and the percentage of spared white matter decreased with time ( $\mathrm{p}<0.05)$ (Fig. 3A and B).

After staining with DAB, the NeuN-positive neurons were brown. There were no significant differences between normal and sham groups in NeuN-positive neuron counts at 1,3 , or 9 weeks. In contrast, the expression of NeuN-positive neurons in the injured cord was remarkably decreased at 9 weeks in the rhBMP-2-treated group $(\mathrm{p}<0.01)$ (Fig. 3C and D).

\section{Histone Modifications and Osteogenic Transcription}

Factors Expressed in the Fibrocartilage Area

The expression of histone $\mathrm{H} 3$ modifications (H3K9ac, $\mathrm{H} 3 \mathrm{~K} 18 \mathrm{ac}, \mathrm{H} 3 \mathrm{~K} 4 \mathrm{me} 3$, and H3K36me3) and osteogenic transcription factors (osterix, Runx2) was examined
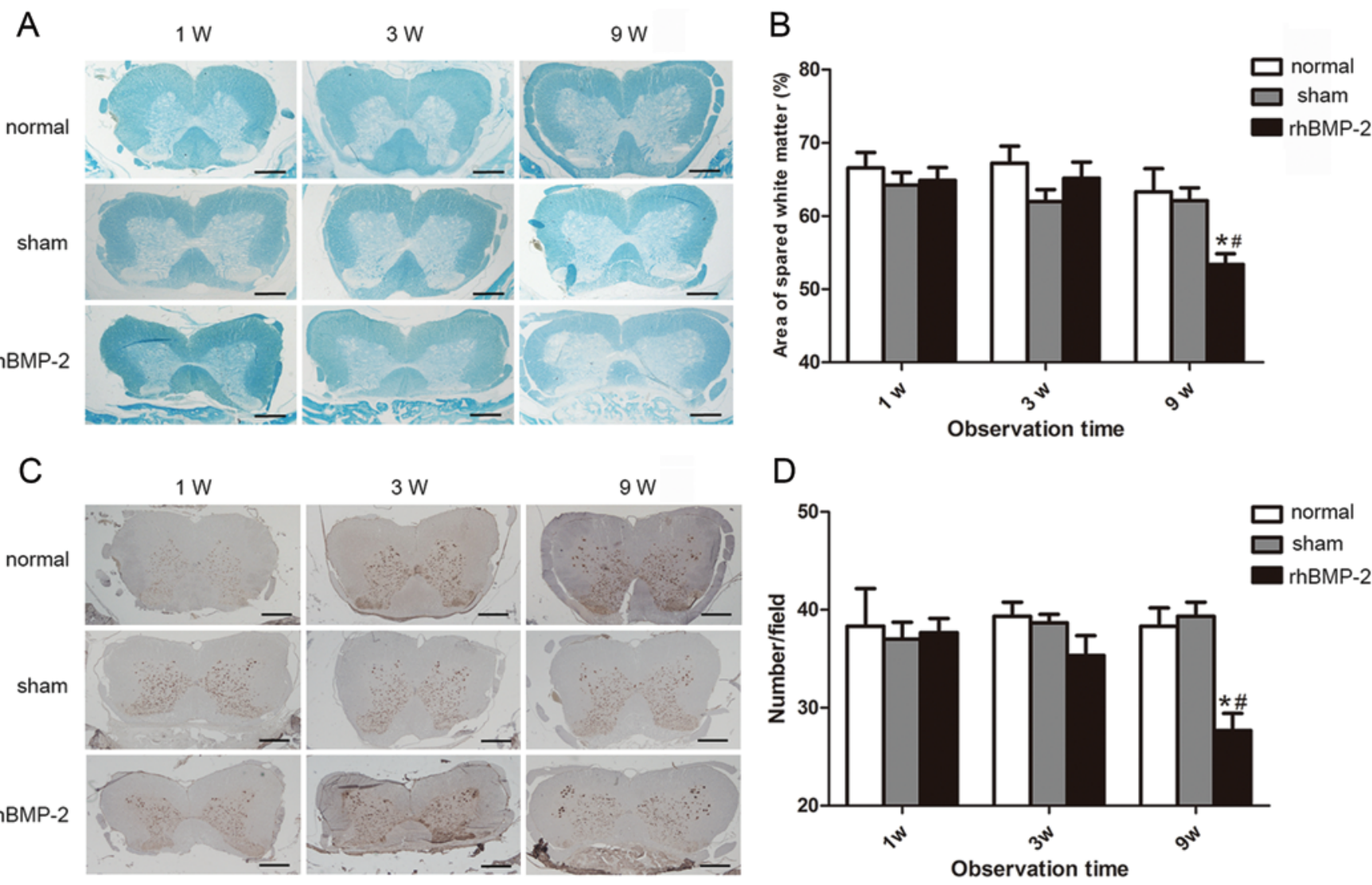

FIG. 3. Comparison of spinal cord cross-sections from the injury epicenter and corresponding cord sections from animals in the sham-operation and normal control groups. A: Photomicrographs of LFB-stained sections showing how the white matter of the dorsal part of the compressed spinal cord became distorted and decreased progressively in the rhBMP-2 group. In the normal and sham-operation control groups, the white matter of the corresponding area of the cord was intact. Bar $=500$ $\mu \mathrm{m}$. B: Quantitative analysis of spared white matter. The mean area of LFB-positive spared white matter in specimens from the rhBMP-2-treated rats $(n=6)$ obtained 9 weeks after surgery was significantly smaller than in specimens from rats in the shamsurgery $(n=6)$ or normal control $(n=3)$ groups as well as specimens obtained from the rhBMP-2 group at 1 week and 3 weeks ( $n$ $=6$ for each time point). ${ }^{*} p<0.05$ for rhBMP-2 group versus normal and sham at 9 weeks. $\# p<0.05$ for rhBMP-2 group at 9 weeks versus 1 and 3 weeks. C: Representative results for anti-NeuN staining of spinal cord cross-sections of the injury epicenter in rhBMP-2-induced OLF and corresponding specimens from the sham-operation and normal control groups. Bar $=500 \mu \mathrm{m} . \quad \mathrm{D}$ : The average number per field (observed at $\times 400$ magnification) of NeuN-positive neurons in the spinal cord. The average number of NeuN-positive neurons in specimens from the rhBMP-2-treated rats $(n=6)$ obtained 9 weeks after surgery was significantly smaller than in specimens from rats in the sham-surgery $(n=6)$ or normal control $(n=3)$ groups as well as specimens obtained from the rhBMP-2 group at 1 week and 3 weeks ( $n=6$ for each time point). ${ }^{*} p<0.01$ for rhBMP-2 group versus normal and sham at 9 weeks. $\# p<0.05$ for rhBMP-2 group at 9 weeks versus 1 and 3 weeks. 


\section{RhBMP-2-induced OLF and associated histone modifications}

with immunohistochemistry. The histone modifications detected in this study indicated different expression levels in each group. In rat OLF samples, the modifications $\mathrm{H} 3 \mathrm{~K} 9 \mathrm{ac}, \mathrm{H} 3 \mathrm{~K} 18 \mathrm{ac}$, and $\mathrm{H} 3 \mathrm{~K} 36 \mathrm{me} 3$ were strongly expressed in the round cells in the fibrocartilage area adjacent to the degenerated irregular ligament. The immunopositivity for $\mathrm{H} 3 \mathrm{~K} 4 \mathrm{me} 3$ was moderate or strong. For the osteogenic transcription factors osterix and Runx2, they were all strongly or moderately expressed in the same area. In the normal and sham-surgery control groups, the nuclei showed no nuclear reactivity (Fig. 4).

\section{Discussion}

In the present study, we successfully induced OLF in the rats. The ossification started at the insertion site in the ligamentum flavum and developed along the ventral surface. The dense elastic fibers became loose and disorganized. Next, chondrocytes appeared between the degenerated fibers and secreted cartilage matrix. The chondrocytes and cartilage were finally replaced by osteoblasts and bone matrix. This process continuously progressed as the observation time was extended. Nine weeks after the operation, normal ligamentum flavum and the transitional fibrous cartilage were almost completely replaced by bone matrix. During this process, the volume of the ossified mass increased at the same time based on micro-CT quantitative analysis ( $\mathrm{p}<0.001)$, and the adjacent spinal cord was obviously distorted and compressed. These characteristics were similar to clinical OLF. ${ }^{22,28}$

There were puzzling features of this OLF model. None of the rats evaluated showed any gait disturbance or paralysis during the observation period, even though the spinal canal was severely encroached and the spinal cord was clearly distorted. The injured cord was stained by LFB and anti-NeuN antibody to detect the content of myelin and the expression of neurons. In comparison with

specimens from the normal and sham-surgery control groups, the specimens from the rhBPM-2 group showed significant decreases in both the content of white matter and number of neurons 9 weeks after surgery, suggesting degenerative changes in the compressed spinal cord. Actually, previous studies using noncommercial BMP-2 (made by the authors) to induce OLF in mice or rabbits also did not produce paralysis, but histological changes were found in those studies that were characteristic of the early pathological condition induced by chronic cord compression. ${ }^{17,23}$ This phenomenon may be due to repair processes within the spinal cord. ${ }^{26}$

Despite the above difference, the pathological features of the ossification process starting from the insertion site of the ligamentum flavum and endochondral ossification suggest that this rhBMP-2-induced OLF was similar to clinical $\mathrm{OLF}^{8}$ and supports the use of this animal model to study OLF pathogenesis in vivo.

Histone modification is a crucial mechanism for the regulation of gene expression and is linked to many fundamental cellular processes, such as stem cell maintenance and differentiation..$^{910,16,21}$ After translation of histone proteins, their $\mathrm{N}$-terminal tails are susceptible to a range of covalent modifications, such as acetylation, methylation, phosphorylation, and ubiquitination. ${ }^{12}$ Histone acetylation and methylation are two of the most investigated modifications. Although immunohistochemical analysis of histone modification cannot examine the relationship between modifications and specific gene activities, this analysis can study these modifications at a cellular level. ${ }^{20}$

The pathogenesis of OLF involves the differentiation of mesenchymal stem cells located in ligamentum flavum into osteoblasts. ${ }^{3,18}$ The ligament tissue is then replaced by bone matrix secreted by osteoblasts. Histone H3 modifications play a critical role in osteoclast differentiation. . $^{67}$ Thus, we suspect modifications of histone $\mathrm{H} 3$ may be involved in regulating the development of OLF.

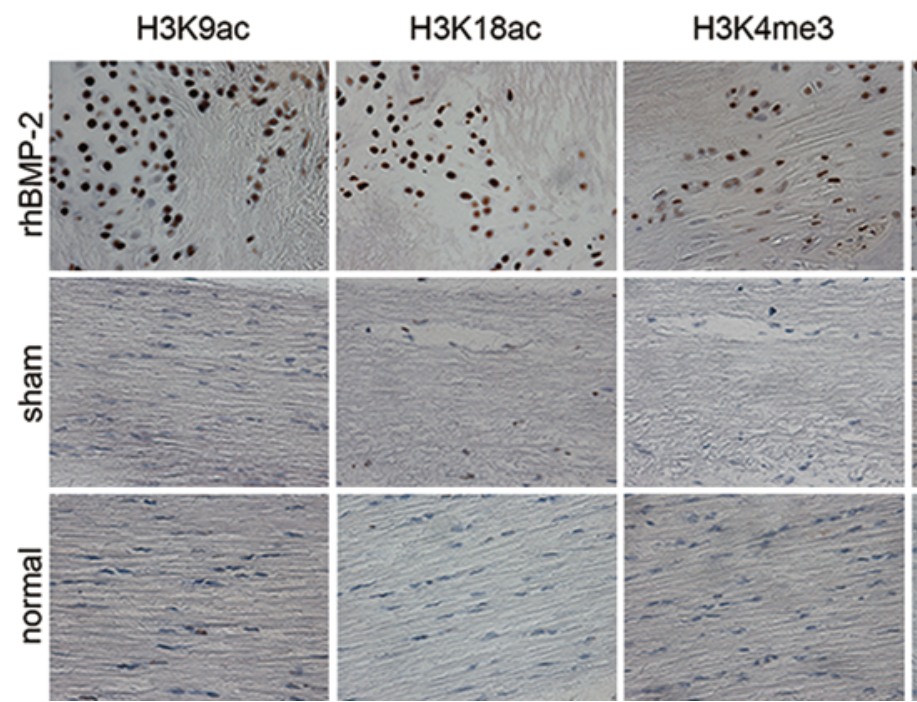

H3K36me3

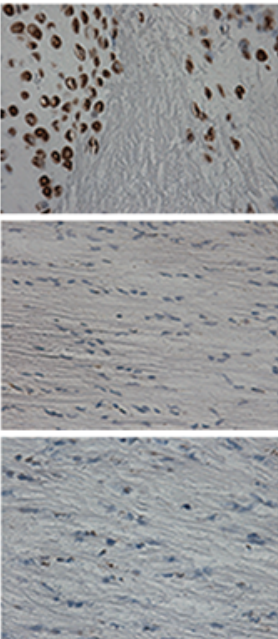

Osterix

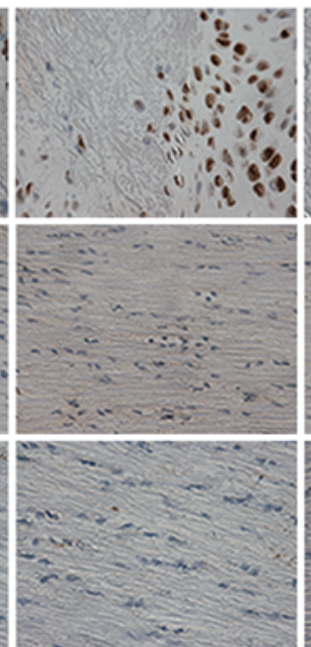

RUNX2

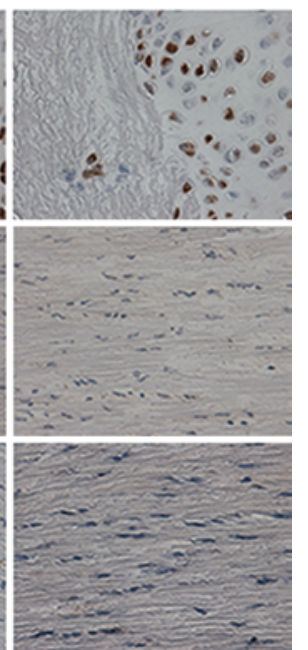

FIG. 4. Representative immunohistochemical results for H3K9ac, H3K18ac, H3K4me3, H3K36me3, osterix, and Runx2 in rhBMP-2-induced OLF and in specimens from the sham-operation group and normal controls. H3K9ac, H3K18ac, and H3K36me3 were strongly positive in the round cells of the fibrocartilage area. H3K4me3 stained with moderate intensity. Osterix and Runx2 were strongly or moderately stained in the same area. In the control groups (sham and normal), the nuclei showed no nuclear reactivity. Original magnification $\times 400$. 
To the best of our knowledge, there has been no report studying the expression of global histone modifications in ossification of spinal ligaments. In the present study, H3K18ac, H3K9ac, H3K4me3, and H3K36me3 were positively expressed in the fibrocartilage tissue, which is in stark contrast to the adjacent degenerated ligament and intact ligamentum flavum, and expression of osterix and Runx 2 was found in the same area. Acetylation can neutralize the positive charge of the histone tails and decrease their affinity for DNA. As a result, the nucleosomal conformation is changed, and this change could increase the accessibility of the transcription regulatory proteins. ${ }^{24}$ Trimethylation of histones H3K4 and H3K36 is associated with the active regions of chromatin., ${ }^{1,4} \mathrm{~A}$ previous study has suggested that osterix and Runx 2 play an important role in the development of OLF. ${ }^{25}$ In this study, these transcription-activating histone modifications and transcription factors localized to the same area. Therefore, we hypothesize that histone modifications (H3K18ac, H3K9ac, H3K4me3, and H3K36me3) create a favorable microenvironment for transcription factors (osterix and Runx2) to access osteogenic genes during ossification. Further studies should be performed to illustrate this relationship.

\section{Conclusions}

In summary, our experiments showed that rhBMP-2induced OLF in rats was similar to clinical OLF. This animal model may be useful for further study of some molecules that are critical during OLF. The compressed spinal cord around the ossification site showed characteristics of a chronic degenerative process. Our experiments suggest that histone modifications are closely related to OLF. Future studies will be necessary to quantitatively analyze the correlation between these modifications and specific osteogenic genes.

\section{Acknowledgment}

We acknowledge the assistance of Dr. Chen Huang (Medical Research Center, Peking University Third Hospital) with applied immunohistochemical techniques.

\section{Disclosure}

This work was supported by the National Natural Science Foundation of China (No. 81071505; No. 30801156) and Ministry of Education of China (No. 200800011041). The authors report no conflict of interest concerning the materials or methods used in this study or the findings specified in this paper.

Author contributions to the study and manuscript preparation include the following. Conception and design: Chen. Acquisition of data: Hou, Sun. Analysis and interpretation of data: Hou, Fan. Drafting the article: Hou. Critically revising the article: all authors. Reviewed submitted version of manuscript: all authors. Approved the final version of the manuscript on behalf of all authors: Chen. Statistical analysis: Hou. Administrative/technical/material support: Fan, Sun. Study supervision: Chen.

\section{References}

1. Bannister AJ, Schneider R, Myers FA, Thorne AW, CraneRobinson C, Kouzarides T: Spatial distribution of di- and tri- methyl lysine 36 of histone $\mathrm{H} 3$ at active genes. J Biol Chem 280:17732-17736, 2005

2. Basso DM, Beattie MS, Bresnahan JC: A sensitive and reliable locomotor rating scale for open field testing in rats. J Neurotrauma 12:1-21, 1995

3. Chen YT, Wei JD, Wang JP, Lee HH, Chiang ER, Lai HC, et al: Isolation of mesenchymal stem cells from human ligamentum flavum: implicating etiology of ligamentum flavum hypertrophy. Spine (Phila Pa 1976) 36:E1193-E1200, 2011

4. Eissenberg JC, Shilatifard A: Histone H3 lysine 4 (H3K4) methylation in development and differentiation. Dev Biol 339:240-249, 2010

5. Fan D, Chen Z, Wang D, Guo Z, Qiang Q, Shang Y: Osterix is a key target for mechanical signals in human thoracic ligament flavum cells. J Cell Physiol 211:577-584, 2007

6. Ge W, Shi L, Zhou Y, Liu Y, Ma GE, Jiang Y, et al: Inhibition of osteogenic differentiation of human adipose-derived stromal cells by retinoblastoma binding protein 2 repression of RUNX2-activated transcription. Stem Cells 29:1112-1125, 2011

7. Guo JJ, Luk KD, Karppinen J, Yang H, Cheung KM: Prevalence, distribution, and morphology of ossification of the ligamentum flavum: a population study of one thousand seven hundred thirty-six magnetic resonance imaging scans. Spine (Phila Pa 1976) 35:51-56, 2010

8. Hoshi K, Amizuka N, Sakou T, Kurokawa T, Ozawa H: Fibroblasts of spinal ligaments pathologically differentiate into chondrocytes induced by recombinant human bone morphogenetic protein-2: morphological examinations for ossification of spinal ligaments. Bone 21:155-162, 1997

9. Jaenisch R, Bird A: Epigenetic regulation of gene expression: how the genome integrates intrinsic and environmental signals. Nat Genet 33 (Suppl):245-254, 2003

10. Karantzali E, Schulz H, Hummel O, Hubner N, Hatzopoulos A, Kretsovali A: Histone deacetylase inhibition accelerates the early events of stem cell differentiation: transcriptomic and epigenetic analysis. Genome Biol 9:R65, 2008

11. Kosaka T, Imakiire A, Mizuno F, Yamamoto K: Activation of nuclear factor $\chi \mathrm{B}$ at the onset of ossification of the spinal ligaments. J Orthop Sci 5:572-578, 2000

12. Kouzarides T: Chromatin modifications and their function. Cell 128:693-705, 2007

13. Lane JM: Bone morphogenic protein science and studies. J Orthop Trauma 19 (10 Suppl):S17-S22, 2005

14. Lang N, Yuan HS, Wang HL, Liao J, Li M, Guo FX, et al: Epidemiological survey of ossification of the ligamentum flavum in thoracic spine: CT imaging observation of 993 cases. Eur Spine J 22:857-862, 2013

15. Lee J, Satkunendrarajah K, Fehlings MG: Development and characterization of a novel rat model of cervical spondylotic myelopathy: the impact of chronic cord compression on clinical, neuroanatomical, and neurophysiological outcomes. J Neurotrauma 29:1012-1027, 2012

16. Melcer S, Hezroni H, Rand E, Nissim-Rafinia M, Skoultchi A, Stewart CL, et al: Histone modifications and lamin A regulate chromatin protein dynamics in early embryonic stem cell differentiation. Nat Commun 3:910, 2012

17. Miyamoto S, Takaoka K, Yonenobu K, Ono K: Ossification of the ligamentum flavum induced by bone morphogenetic protein. An experimental study in mice. J Bone Joint Surg Br 74:279-283, 1992

18. Murata H, Tanaka H, Taguchi T, Shiigi E, Mizokami H, Sugiyama T, et al: Dexamethasone induces human spinal ligament derived cells toward osteogenic differentiation. J Cell Biochem 92:715-722, 2004

19. Nakase T, Ariga K, Yonenobu K, Tsumaki N, Luyten FP, Mukai Y, et al: Activation and localization of cartilage-derived morphogenetic protein-1 at the site of ossification of the ligamentum flavum. Eur Spine J 10:289-294, 2001

20. Nakazawa T, Kondo T, Ma D, Niu D, Mochizuki K, Kawasaki 


\section{RhBMP-2-induced OLF and associated histone modifications}

$\mathrm{T}$, et al: Global histone modification of histone $\mathrm{H} 3$ in colorectal cancer and its precursor lesions. Hum Pathol 43:834-842, 2012

21. Noer A, Lindeman LC, Collas P: Histone H3 modifications associated with differentiation and long-term culture of mesenchymal adipose stem cells. Stem Cells Dev 18:725-736, 2009

22. Ono K, Yonenobu K, Miyamoto S, Okada K: Pathology of ossification of the posterior longitudinal ligament and ligamentum flavum. Clin Orthop Relat Res (359):18-26, 1999

23. Saito H, Mimatsu K, Sato K, Hashizume Y: Histopathologic and morphometric study of spinal cord lesion in a chronic cord compression model using bone morphogenetic protein in rabbits. Spine (Phila Pa 1976) 17:1368-1374, 1992

24. Sterner DE, Berger SL: Acetylation of histones and transcription-related factors. Microbiol Mol Biol Rev 64:435-459, 2000

25. Uchida K, Yayama T, Cai HX, Nakajima H, Sugita D, Guerrero AR, et al: Ossification process involving the human thoracic ligamentum flavum: role of transcription factors. Arthritis Res Ther 13:R144, 2011

26. Wang J, Rong W, Hu X, Liu X, Jiang L, Ma Y, et al: Hyaluronan tetrasaccharide in the cerebrospinal fluid is associated with self-repair of rats after chronic spinal cord compression. Neuroscience 210:467-480, 2012

27. Yasui T, Hirose J, Tsutsumi S, Nakamura K, Aburatani H, Tanaka S: Epigenetic regulation of osteoclast differentiation: possible involvement of Jmjd3 in the histone demethylation of Nfatc1. J Bone Miner Res 26:2665-2671, 2011
28. Yayama T, Uchida K, Kobayashi S, Kokubo Y, Sato R, Nakajima $\mathrm{H}$, et al: Thoracic ossification of the human ligamentum flavum: histopathological and immunohistochemical findings around the ossified lesion. J Neurosurg Spine 7:184-193, 2007

29. Yokosuka K, Park JS, Jimbo K, Yoshida T, Yamada K, Sato K, et al: Immunohistochemical demonstration of advanced glycation end products and the effects of advanced glycation end products in ossified ligament tissues in vitro. Spine (Phila Pa 1976) 32:E337-E339, 2007

30. Zhong ZM, Chen JT, Zhang Y, Zha DS, Lin ZS, Zhao CY, et al: Growth/differentiation factor-5 induces osteogenic differentiation of human ligamentum flavum cells through activation of ERK1/2 and p38 MAPK. Cell Physiol Biochem 26:179-186, 2010

Manuscript submitted April 2, 2013

Accepted April 30, 2014.

Please include this information when citing this paper: published online June 20, 2014; DOI: 10.3171/2014.4.SPINE13319.

Address correspondence to: Zhong-Qiang Chen, M.D., Department of Orthopedics, Peking University Third Hospital, 49 North Garden Rd., Haidian District, Beijing 100191, China. email: puh3_ czq@yahoo.com. 